\title{
PRELIMINARY DESIGN CONCEPT OF A SUPERCRITICAL REACTOR USING AVAILABLE RESOURCES
}

\author{
NE 471 \\ DEPARTMENT OF NUCLEAR ENGINEERING \\ THE UNIVERSITY OF TENNESSEE, KNOXVILLE \\ FALL SEMESTER, 1993
}

AUTHORS: Edward L. Churnetski

Vaclav Hoyny

Baishali Ray Chaudhuri

Andreas Taprantzis

Ahmet Yavas 


\section{TABLE OF CONTENTS}

1.0 Summary 3

2.0 Introduction 5

$\begin{array}{lll}3.0 & \text { Prior Work } & 10\end{array}$

4.0 Description of Work Performed 11

4.1 Technical Approach 12

$\begin{array}{lll}4.2 & \text { Results } & 15\end{array}$

4.2.1 Limiting Calculations 15 •

$\begin{array}{ll}\text { 4.2.2 Fuel Lumping Studies } & 17\end{array}$

$\begin{array}{ll}\text { 4.2.3 Baseline Designs } & 18\end{array}$

4.2.3.1 Rod Geometry - Triangular Pitch 18

4.2.3.2 Discrete Slugs - BCC Lattice 20

$\begin{array}{ll}\text { 4.2.4 Alternative Moderators } & 22\end{array}$

$\begin{array}{lll}4.2 .5 & \text { Reflectors } & 24\end{array}$

$\begin{array}{ll}\text { 4.2.6 Cladding Poisoning } & 24\end{array}$

4.2.7 Temperature/Multiplication Relationships 25

$\begin{array}{lll}5.0 & \text { Conclusions } & 27\end{array}$

$\begin{array}{lll}6.0 & \text { Future Work } & 28\end{array}$

Appendix

References 


\subsection{Summary}

During the Fall 1993 semester, a project was initiated within the Nuclear Engineering Department of the University of Tennessee with the objective of developing a design for a subcritical reactor with maximized multiplication factor using materials currently available. Such a device, if constructed, would serve as a teaching tool for the Department of Nuclear Engineering, which in recent years has experienced a severe curtailment in access to operating research reactors.

For the purposes of this study, the available material inventory consists primarily of $\mathbf{5 8 0 0}$ aluminum clad cylindrical uranium metal fuel slugs of uncertain isotopic assay; various sources place the probable assay in the "depleted" (less than $0.71 \% \mathrm{U}^{235}$ ) to natural range. In order for design work to proceed in parallel with attempts to determine assay, the fissile content of the fuel was treated as a variable in calculations.

Design work was conducted as a large number of computer calculations, with trial pile configurations based on fundamental nuclear engineering principles, in an effort to maximize multiplication factor through fuel element geometry, moderator type, fissile/moderator ratio, and reflector character. The principal objective of the design group for the early phase of this project was to present several possible "baseline" reactor designs and identify directions for improvements. For the sake of calculational ease, the cores analyzed to date have been of nearly cubic shape. The SCALE CSAS25 software which runs KENO.Va, a Monte Carlo code, was 
used for all neutronics calculations.

The baseline reactors resulting from work to date are cuboidal in shape and graphite reflected. Two types of fuel element geometries are proposed, a typical triangular pitch rod lattice and an arrangement of discrete fuel slugs placed in a lattice corresponding to body centered cubic packing. The latter arrangement provides slightly higher multiplication factors than the former. Calculations were performed for both graphite and heavy water moderation with heavy water moderation producing considerably higher multiplication factors, as expected. In general, the maximum $\mathrm{k}_{\text {eff }}$ for the reactors are in the range of 0.5 to 0.9 , well subcritical, except in the cases of the extreme possible values of fuel assay where critical configurations are possible. In these cases, designs with reduced fuel loading are recommended to assure a subcritical multiplication factor. 


\subsection{Introduction}

In recent decades, many socioeconomic factors have coalesced to effectively hinder, through government intervention at all levels, the construction and operation of commercial power, defense, and research nuclear reactors. When viewed in the aggregate, the difficulties imposed on the construction of new reactors, the age of existing research reactors, and the regulatory hurdles imposed by oversight bodies portend a generally grim future for reactor-based nuclear research in the United States. In particular, the Nuclear Engineering Department at the University of Tennessee (UT), which for most of its history had access to the many research reactors operated by Oak Ridge National Laboratory, is now relegated to very limited access to the sole reactor extant at Oak Ridge, and the future availability of this reactor is questionable.

The availability of a research reactor is desirable for the maintenance of a high quality nuclear engineering teaching program such as that currently existing at UT. Although nuclear engineering is a rather theory intensive discipline, which has historically depended very heavily on modeling, the validity of any theory or model must still rely on empirical verification. Therefore, there is an understandable desire on the part of the teaching staff at UT to identify alternatives to the reactors in Oak Ridge for the purposes of practical instruction.

In this context, a design problem was assigned to the Fall 1993 Nuclear Engineering 471 class which, paraphrasing, charged the class to design, with available materials, a subcritical pile which maximized $k_{\text {eff }}$ The available materials consist primarily of a substantial quantity of 
uranium and a large amount of reactor grade graphite. A verbal history indicates that the uranium was supplied to UT in the late 1950's or early 1960's by the AEC, a Department of Energy predecessor, for use as fuel slugs in highly subcritical experiments. These slugs are cylinders approximately $11 \mathrm{~cm}$ long by $3.0 \mathrm{~cm}$ in diameter (which includes a $2 \mathrm{~mm}$ aluminum cladding). Density measurements suggest that the uranium is in the metallic form.

Since all of the fuel material can not be directly inspected, for the purposes of this study the number of available slugs was assumed to be 5800 , the number believed by the Nuclear Engineering Department to be stored in the vault on the first floor of Pasqua Engineering Building. A direct counting of the slugs was not practicable, but there is some indication that somewhat more than 5800 slugs may actually be stored. However, these quantities are large enough that the use of the different masses in calculations causes no qualitative, and little quantitative, difference in behavior. Since the possibility exists for the availability of additional uranium from other universities or the DOE, the conservative lower limit 5800 count is deemed appropriate for the early stages of a design study.

The absence of information on the actual isotopic assay of the uranium in the fuel slugs is much more of a hinderance to the design effort than uncertainties in quantity. Apparently, the assay of the material is reported as either natural or depleted on various documents, allowing the $\mathrm{U} 235$ assay to vary between $0.71 \%$ to, perhaps, as low as $0.2 \%$. Since this range in assay results in large differences in the calculated values of both $k_{\propto}$ and $k_{\text {eff }}$ (over the low assay end 
of this range, simple hand calculations suggest that, even in an ideal system, core multiplication will be far less than unity, while there is quite clear empirical evidence that at the high end of the assay range criticality is easily attained) the approach taken by the design team, for the work performed to date, was to treat fissile content as a variable and design reactors for several possible assays. The probable assay is believed by the design team to be on the order of 0.3 to $0.4 \%$, so somewhat greater calculational effort was expended in this range. For calculations where it was not deemed worthwhile to cover the entire range of possible assays, a value of $0.3 \%$ was assumed. A request has been made, and approved, for assistance from the DOE in determining the composition of the UT fuel slugs. Efforts to transfer a small number of samples to a DOE contractor-operated facility for analysis are underway.

A basic assumption in this work is that, due to fiscal and regulatory constraints, little if anything can be done to alter the composition or configuration of the fuel slugs. Therefore, the proposed reactor designs employ a fuel geometry and composition generally achievable with the use of unaltered fuel slugs. (Some limited work, mainly of theoretical interest, was performed in evaluating the effects of fuel lumping and stripping of the aluminum cladding). Little consideration is given to limitations on the availability of graphite moderator since this material is readily available commercially and can, possibly, even be obtained from excess stocks at other institutions.

Recognizing the near perfection of deuterium as a moderator material, all design cases were also analyzed with heavy water $\left(\mathrm{D}_{2} \mathrm{O}\right)$ moderation to examine the extremes of the 
multiplication envelope. Although it is unlikely, due to the high cost of deuterium, that sufficient heavy water can be purchased for construction of the proposed reactor, the Canadian and US governments have stockpiles of heavy water for which a loan agreement might be explored. There are precedents for the loan of government-owned stockpile material for academic purposes.

As part of the design effort, beryllium, beryllium oxide, liquid oxygen, and liquid helium -4 have been evaluated as alternative moderators. The investigators' rational for examining these materials is the potential for improved performance over graphite but at much lower cost than deuterium-containing substances. Limited comparisons of various non-deuterated reflector materials were also performed. The low effort on reflector evaluation resulted from early calculations which yielded, for the core dimensions of interest, nonleakage probabilities of greater than $90 \%$ and the observed similarities in the effectiveness of good reflector materials. A brief examination of the effects of temperature on multiplication factor has also been performed in an attempt to assess the potential value of operating a reactor at cryogenic temperatures.

With the decision to retain as invariant the configuration of the fuel slugs, freedom in designing the pile is drastically reduced. Additionally, the neutronics software employed make difficult the analysis of extremely irregular or free-form surface geometries. With these constraints, an approach focused on two types of core configurations, the "baseline" designs, was adopted by the design group. The first pile configuration analyzed, due to its relative 
simplicity and known effectiveness, was a triangular pitch rod lattice. Recognizing that a more uniformly dispersed spacial arrangement of the fuel should enhance the overall neutron economy of the core, the second baseline design examined was a body centered cubic lattice of individual slugs. The optimum pitch was determined for each enrichment and geometry by performing unit cell calculations with complete return boundary conditions.

Although an overall core shape approximating a sphere is preferable in order to minimize neutron leakage, calculational and resource limitations lead to standardization of the baseline designs to approximate cubes (cuboids). The above stated assumptions on fuel limitations dictated the overall dimensions of the cores, except in the case of the very highest assays in combination with heavy water moderation, where the optimum pitch was employed but the entire available fuel inventory was not used in order to assure a subcritical configuration. All core designs analyzed were assumed to be reflected by two meters of graphite on all sides.

No structural analyses were performed for either of the core types investigated. The presumption is that, in the case of the graphite, the mechanical strength of the moderator would be more than suf.icient to support the fuel load in any likely configuration. In the case of heavy water moderation, some type of support lattice would obviously be required for the slugs. The nature of this support might be, for example, an aluminum or zirconium alloy structure, or a hanging arrangement employing high strength polyolefin fiber. Absorption from support materials are not accounted for in the neutronics calculations. No thermal hydraulic analysis is warranted for the systems under consideration. 
The principal results from this design study are in the form of graphical relationships between effective multiplication factor $\left(k_{\text {eff }}\right)$ and fuel assay for the two baseline graphite reflected cuboidal core configurations described above, with a fuel constraint of approximately 5800 aluminum clad uranium metal slugs and with either graphite or deuterium oxide as moderators.

The pile neutronics calculations were performed with the CSAS25 module of the SCALE system which employs the KENO-Va Monte Carlo code for multiplication factor calculations and the BONAMI and NITAWL codes for cross section processing. Default values were routinely used for input parameters with the exception of reflector specifications. The majority of the calculations supporting this study were performed on the IBM 3090 computer at UT.

\subsection{Prior Work}

This is the first progress report on this project. The design group is aware of no prior similar efforts chartered by the Nuclear Engineering Department at UT. 


\subsection{Description of Work Performed}

The design of the subject core is based primarily on the results of a large number of computer "experiments" in which various pile parameters and geometries were modeled and observed, and an index of performance, either the infinite multiplication factor $\left(\mathbf{k}_{\alpha}\right)$ or $\mathbf{k}_{\mathrm{eff}}$, was obtained. A relatively minor amount of nuclear engineering theory is employed to design the configurations used as input to the SCALE Monte Carlo codes. The results from the calculations are used to identify maxima in the index of performance and hence the optimum design parameters for the system. This is a rather brute force method, but is deemed to be the best approach by the design group since it is, in principle, equivalent to "constructing" full reactors for experiments, and computational resources were, for practical purposes, unlimited.

In the majority of the calculations run, assay appeared as a variable, which suggested a convenient form for the calculational matrix and division of labor amongst the design group. Typically, pitch and assay appeared as variables with $\mathbf{k}_{\propto}$ forming the response surface. 


\subsection{Technical Approach}

Before effort was expended on Monte Carlo computations, decisions were made by the design group regarding the boundaries of the core parameter envelope. Principal among these is the decision to limit analysis to relatively simple cuboidal shapes. Consideration of only the triangular pitch rods and body centered cubic lattice fuel configurations likewise severely bounds the design. With the decisions on geometry made, a modest amount of computational work was performed to examine the effects of various moderators on core multiplication. This was followed by a brief evaluation of reflector effectiveness.

The results of these preliminary calculations are the selection of graphite and heavy water as moderators and graphite as reflector; this completely specifies the reactor composition while the decisions made on shape and fuel configuration fix core geometry. Only dimensional specifications of the cores remain to be determined, a process which to date has been accomplished in a methodical fashion by first determining the optimum pitch for a given assay and fuel configuration and then calculating the overall dimensions of the core with the constraint on available fuel.

Optimum pitch calculations were performed in all cases by identifying a cuboidal unit cell of the core geometry being evaluated, applying appropriate boundary reflection conditions, and performing computations to determine the multiplication versus pitch relationship. The cell boundaries are; in the case of infinite dimensions, flux symmetry planes, so the use of a 
complete return reflectance at these boundaries is sufficient to ensure that the flux in the finite unit cell being examined is indistinguishable from any other cell in an infinite core constructed from these cells. In the case of a body centered cubic arrangement of fuel slugs, which is identical, symmetrical, and infinite in three dimensions, the boundary conditions employed are specular reflection on all faces. However, the boundary conditions used in analysis of the rod lattice unit cells, which have a lower degree of symmetry than the body centered cubic, accounted for leakage from the ends of the finite cell axis (rod axis direction) in an "infinite" core by imposing a real material reflector condition in this direction and specular reflection on the perpendicular symmetry planes; the $\mathbf{k}_{\alpha}, \operatorname{li}_{n} \mathrm{n}$ the rod lattice calculations is therefore probably better described as a pseudo- $\mathrm{k}_{\alpha}$. The configurations of the rod lattice and body centered cubic lattice unit cells are shown in figures 1 and 2, respectively. Examples of typical input codes for unit cell calculations based on triangular pitch rod geometry and fuel slugs set in a body centered cubic lattice are presented in appendix $\mathbf{A}$.

With the relationship between $\mathbf{k}_{\alpha}$ and pitch known, the finite cores can be designed by arranging units cells so as to achieve an approximately cubic core with the available fuel. In all cases, in the interest of neutron economy, the optimum pitch is used in the finite core. Finally, specification of a two meter graphite reflector allows calculation of $k_{\text {eff }}$ for the reactors.

The auxiliary calculations, which included the evaluation of alternative moderator materials, the effectiveness of common reflectors, and the determination of the poisoning effects of the aluminum cladding, were accomplished by calculations based on a body centered cubic 


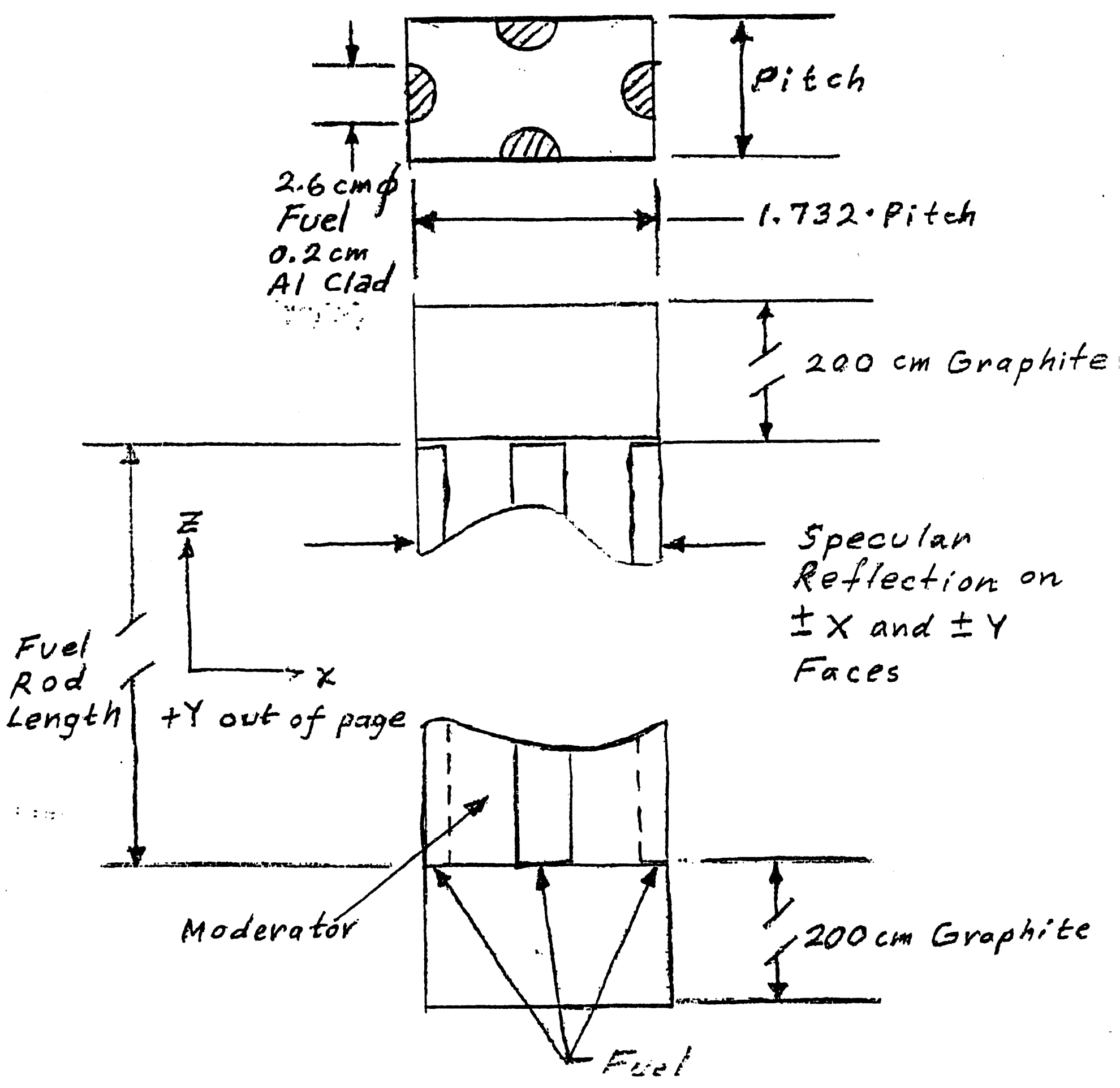

Fig 1: Unit cell For Triangular Pitch Rod Lattice. 

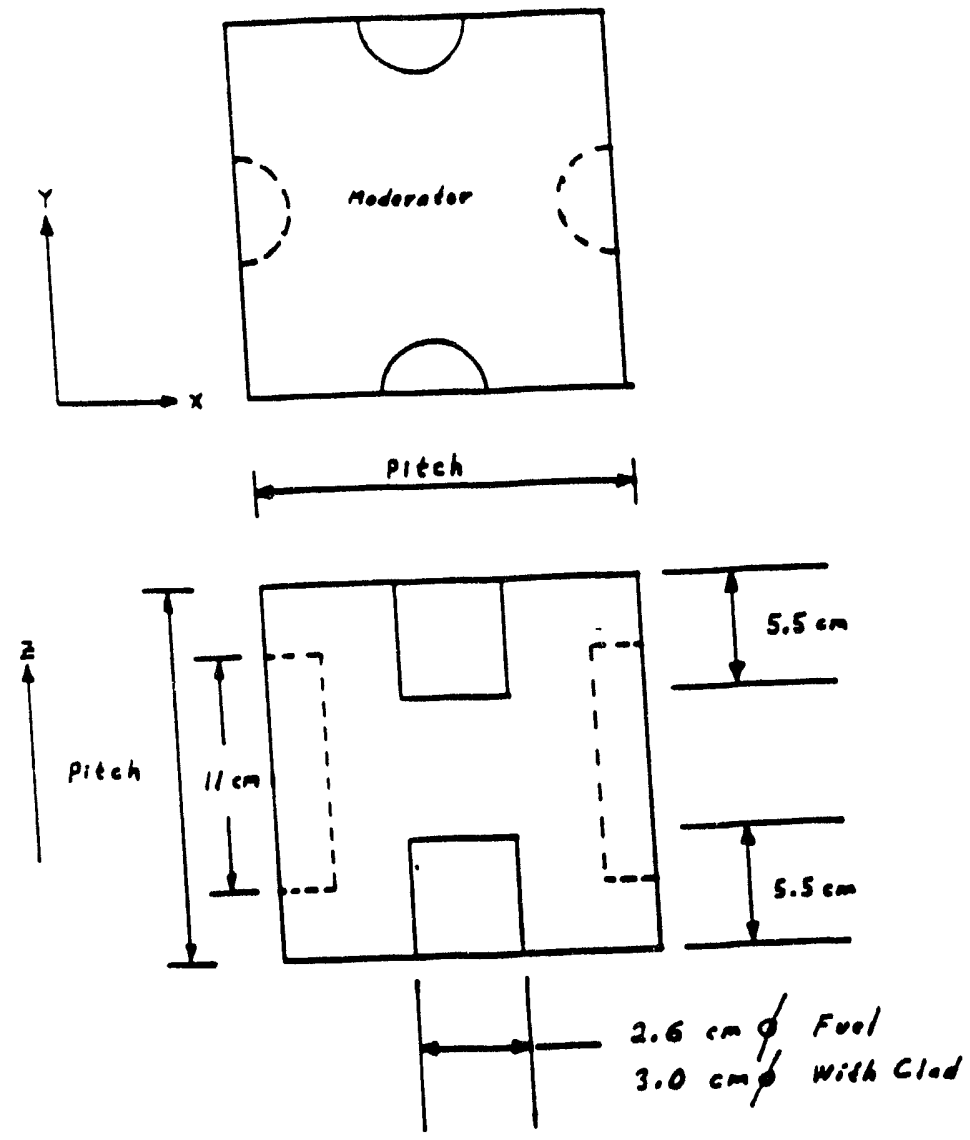

Fig2. Body Centered Cubic Uait Cell 
unit cell.

The unit cell definition for the rod lattice geometry used in this study has a non-zero leakage which depends on the quantity of available fuel, while the body centered cubic unit cell has no such relationship to the fuel constraint. Therefore, the body centered cubic geometry was chosen for all auxiliary calculations. 


\subsection{Results}

Presented below are the results of supporting calculations and the design parameters, as functions of fuel assay, of the baseline systems. Brief explanations are provided of the theory, calculational procedures and assumptions employed.

\subsubsection{Limiting Cases (Simple Calculations)}

Often, an instructive exercise is the examination of limiting cases, by simple analytical methods, in order to obtain an idea of what is possible and/or probable and have a basis of comparison for more refined calculations. Before beginning Monte Carlo calculations for the design effort, the classic four factor formula (eqn. 1) for determination of $k \propto$ in a thermal system was employed to assess the approximate range of the multiplication factor versus assay envelope.

$$
k_{\alpha}=\epsilon \eta \mathrm{fp}
$$

where: $\epsilon=$ fast fission factor

$$
\begin{aligned}
& \eta=\text { reproduction factor } \\
& \mathbf{f}=\text { thermal utilization factor } \\
& \mathbf{p}=\text { resonance escape probability }
\end{aligned}
$$

As a limiting case, the resonance escape probability was assumed to be unity, absorption 
by moderator nuclei was ignored, and the commonly used average value of 1.05 was used for the fast fission factor. With these assumptions, the relationship between assay and $\mathrm{k} \propto$ can be calculated and is displayed in Fig 3. An obvious conclusion which can be drawn from the behavior of $\mathbf{k}_{\infty}$ is that at the low end of the potential assay range there is no possibility of achieving a critical configuration. Furthermore, factoring in reasonable resonance escape and nonleakage probabilities and moderator absorption makes criticality seem unlikely at all but the highest assays. This is indeed what is observed in the results of monte carlo calculations. 


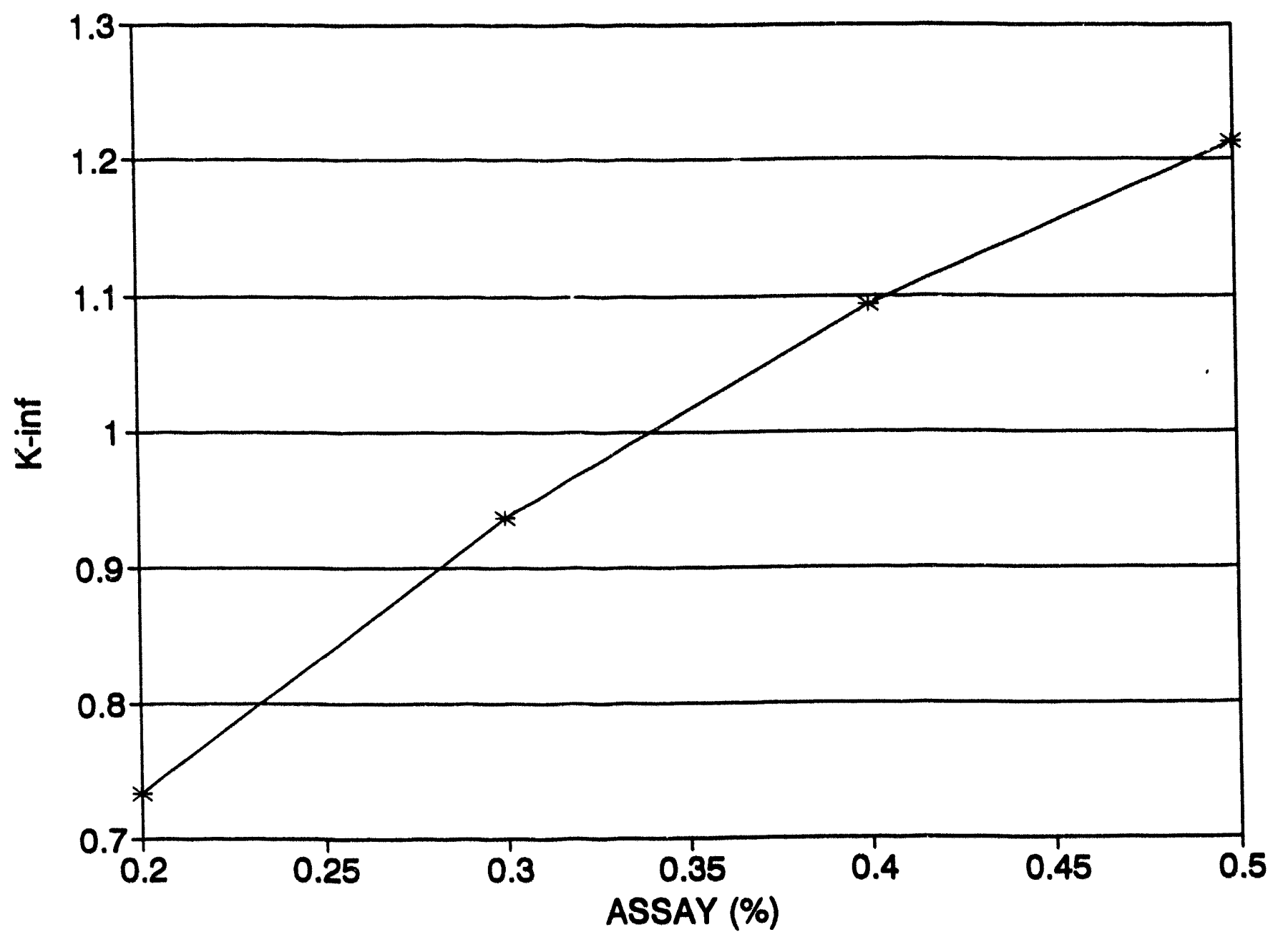

Fig 3. $K_{\propto}$ VS ASSAY as predicted by Eqn 1 


\subsubsection{Fuel Lumping Studies}

Table 1 shows the effects of fuel lumping on the multiplication factor in a graphite moderated, semi-infinite, fuel limited (5800 slugs) array of rods (a pseudo $k \propto$ ). Different diameters of fuel rods and different pitch/diameter ratios were considered in an effort to maximize the multiplication factor. The diameters were $2,2.6,3$ and $5 \mathrm{~cm}$ while the pitch/diameter ratios were $3,4,5,6,7,8$ and 10 . All these cases were tried for a fuel assay of $0.3 \%$.

TABLE 1. $K_{\alpha}$ FOR VARIOUS FUEL DIAMETERS AND PITCH/DIAMETER RATIOS

\begin{tabular}{|l|l|l|l|l|l|l|l||}
\hline Fuel & \multicolumn{6}{|l||}{ Pitch/Diameter Ratio $:$} \\
Rod \\
Diamete
\end{tabular}

The graphs resulting from the above table are shown in Fig 4. From the figure it is evident that in order to maximize $k_{\text {eff }}$, the pitch-diameter ratio needs to be increased for larger diameters and vice versa. Also, the optimum diameter lies between $3 \mathrm{~cm}$ and $5 \mathrm{~cm}$. Diameters less than $3 \mathrm{~cm}$ will result in lower multiplication than the diameters considered, whatever the pitch/diameter ratio is. 


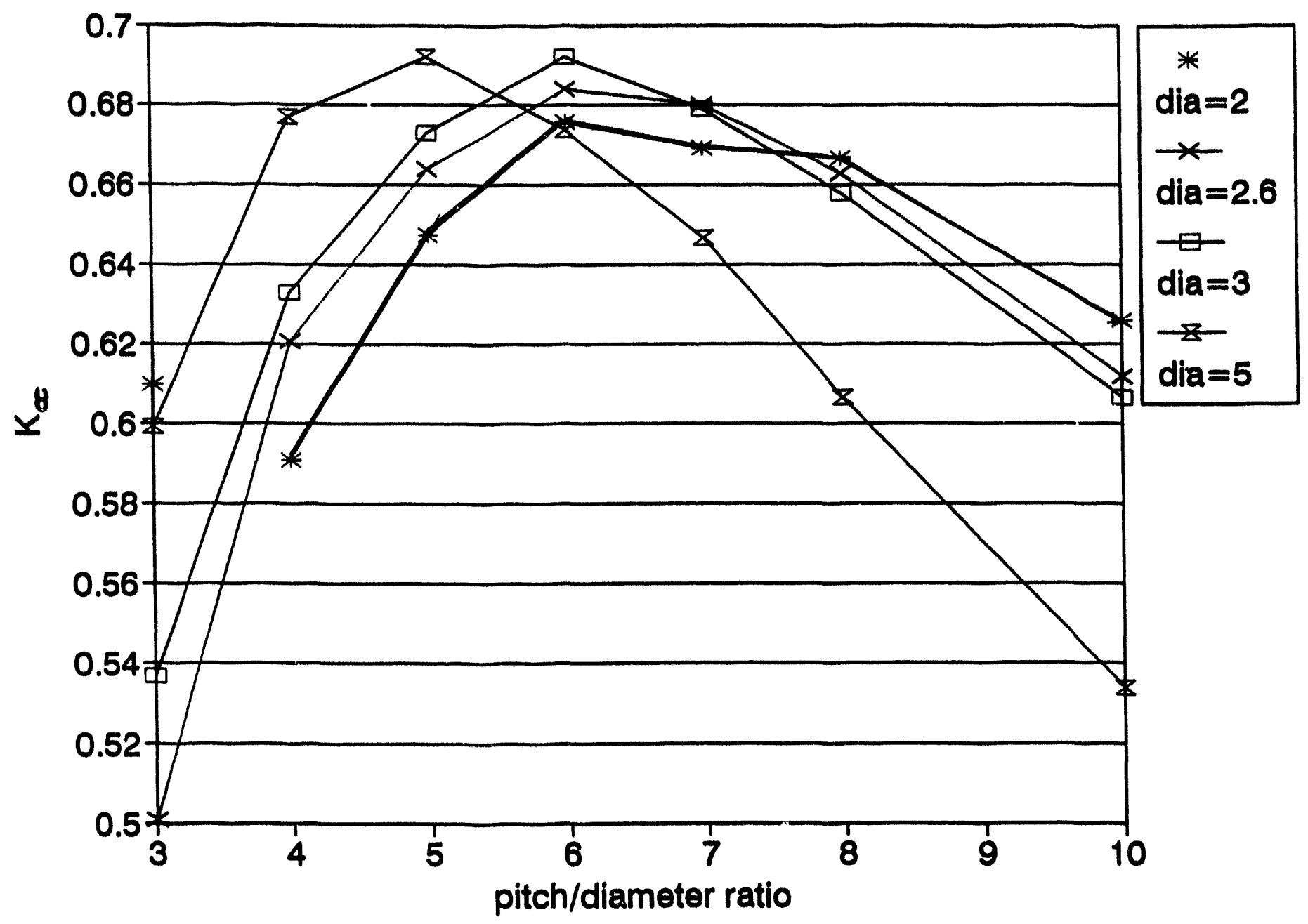

Fig 4. $K_{\infty}$ MATRIX, graphite mod, semi-inf, array of fuel rods 


\subsubsection{Baseline Designs}

Due to the difficulty in altering the shape and/or composition of the existing fuel slugs, the decision was made by the design group to design systems using the unaltered slugs. Two core configurations were chosen as baseline cases, a triangular pitch rod lattice and an arrangement of the fuel slugs in a body centered cubic lattice. Both of these designs make efficient use of the moderator volume, but the body centered cubic arrangement would be expected to be somewhat superior in performance due to the three axis symmetry of the system and consequent more efficient moderator usage. The proposed baseline designs are reflected by two meters of graphite. Multiplication factors are presented for both graphite and heavy water moderation.

\subsubsection{Rod Geometry -- Triangular Pitch}

The nuclear analysis and design of a reactor core is highly dependent on other areas of core design including thermal-hydraulic design, structural analysis, economic performance, and so on. The criteria for a design effort are quite varied, including considerations of performance, reliability, economics, and safety. These criteria are frequently contradictory in nature, so they require optimization. 
One of the baseline designs considered herein is a triangular pitch arrangement of rods.

The parameters that reflect fuel constraint of $\mathbf{5 8 0 0}$ slugs and illustrate this design are:

$$
\begin{aligned}
& \mathrm{L}=(27.709) \mathrm{P} 0.667 \\
& \mathbf{n x}=\mathrm{L} / \mathrm{P} \\
& \text { ny } \quad=\quad L /(1.732) P \\
& \text { Where: } \mathrm{L}=\text { length } \\
& \mathrm{P} \quad=\quad \text { Pitch (the free variable) }
\end{aligned}
$$

In this design, we first found the optimum pitch. With the best reflector and the optimum pitch, we determined $k_{e f f}$ for the pile as a function of assay using both graphite and $\mathrm{D}_{2} \mathrm{O}$ as moderator. The results are given in Fig 5 and Fig 6 respectively. 


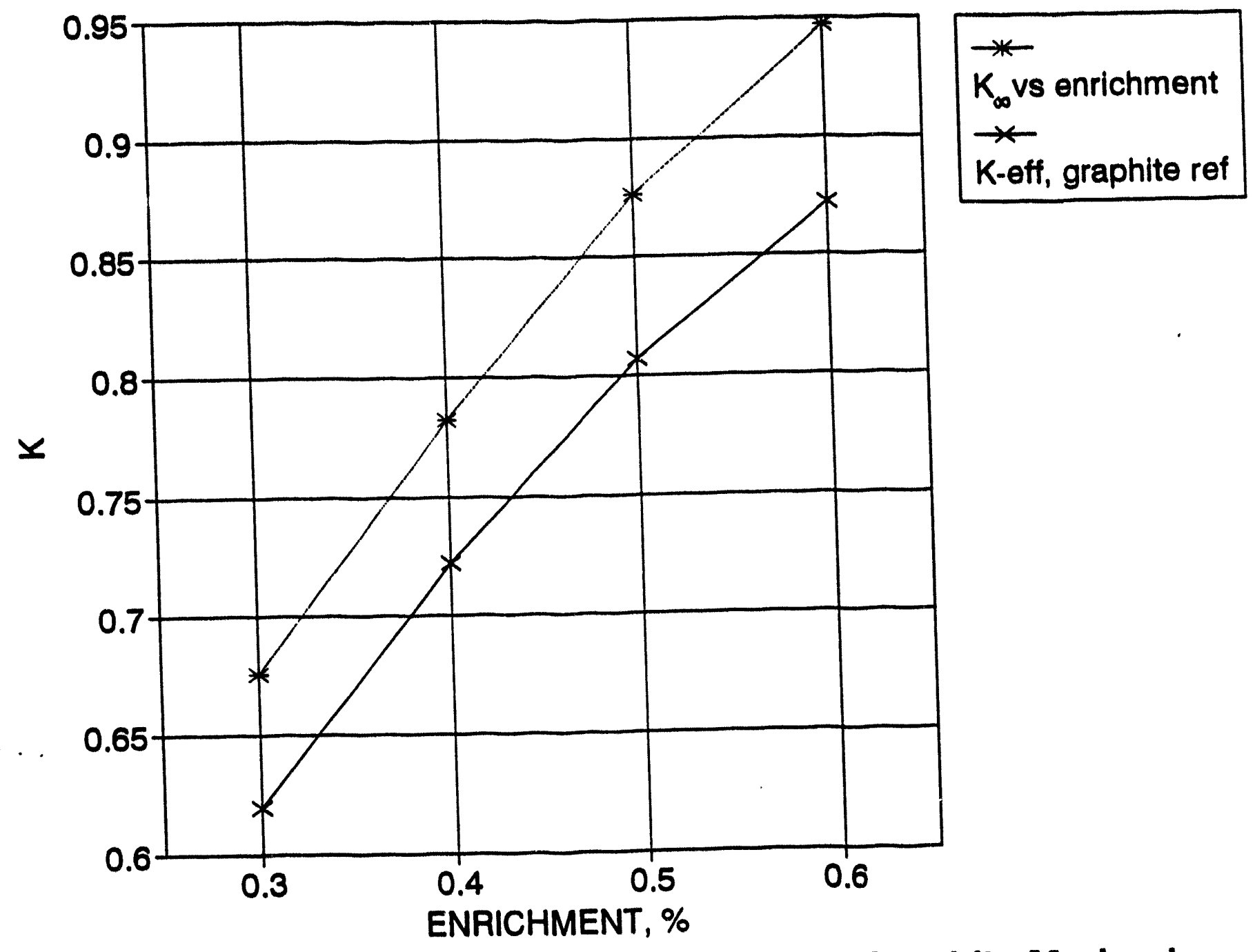

Fia 5. K VS ENRICHMENT. Rod Lattice. Graphite Moderator 


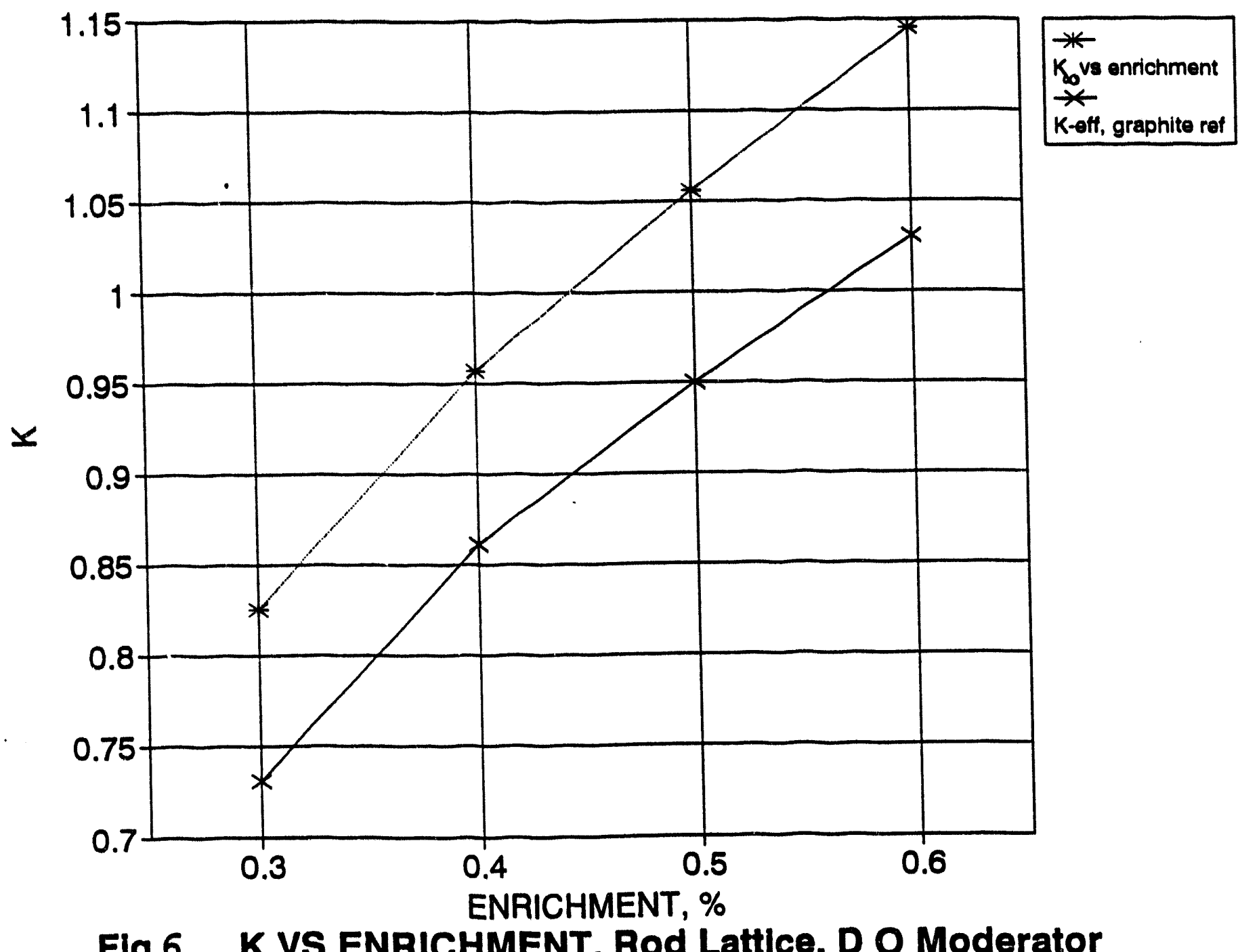

Fig 6 K VS ENRICHMENT, Rod Lattice, $D_{2} O$ Moderator 


\subsubsection{Discrete Slugs - BCC Lattice}

It is obvious that moderator should be used in such a way that fast neutrons born in fission can reach the moderator quickly while moving in any direction. Therefore spheres of uranium uniformly distributed in the moderator would be the best possible arrangement. But in compliance with the constraint of available resources, we have considered discrete slugs of uranium with diameter $3 \mathrm{~cm}$ and length $110 \mathrm{~cm}$ ( including cladding ). Therefore we have decided to separate uranium slugs in the reactor with moderator creating a sort of approximation of the ideal arrangement.

The arrangement outlined above has been modeled using the body centered cubic (BCC) lattice, i.e. a kind of both-horizontal-and-vertical triangular-pitch-array design. A basic unit of the model is the cubic cell shown in Fig 2. These cells have then been arranged in a cuboidal array of $14 \times 14 \times 15$ cells as this number of cells assures maximum utilization of the available inventory of slugs which is roughly 5800 pieces. The resulting core has then been, as in previous cases, reflected from all sides by graphite which has proved to be the best reflector evaluated in this study.

The described design represents a close approximation of the most effective design possible while keeping the original form of the uranium slugs. There exists an untested possibility that another pitch in the direction of slug axes (vertical) than the one chosen - i.e. the same pitch in vertical direction as the one proved optimal in the horizontal direction ( cf. previous sections) 
would be more effective. This option has not been tested due to the lack of time as it would need another set of extensive calculations when looking for optimum pitches separately in both horizontal and vertical directions. More sophisticated packing arrangements such as face centered cubic might also provide higher multiplication factors. The results obtained for the body centered cubic core arrangement are shown in Figures 7 and 8 for graphite and heavy water moderators respectively.

The comparison of the results, both for the unit cell and for the whole reactor, with the results for the rod geometry described in previous section has fulfilled the expectations. Body centered cubic design gives slightly greater values of the $k_{\propto}$ and $k_{\text {eff }}$ respectively. The difference, however, is small (about 2 to $4 \%$ for the cell, and about 0.5 to $2 \%$ for the array). 


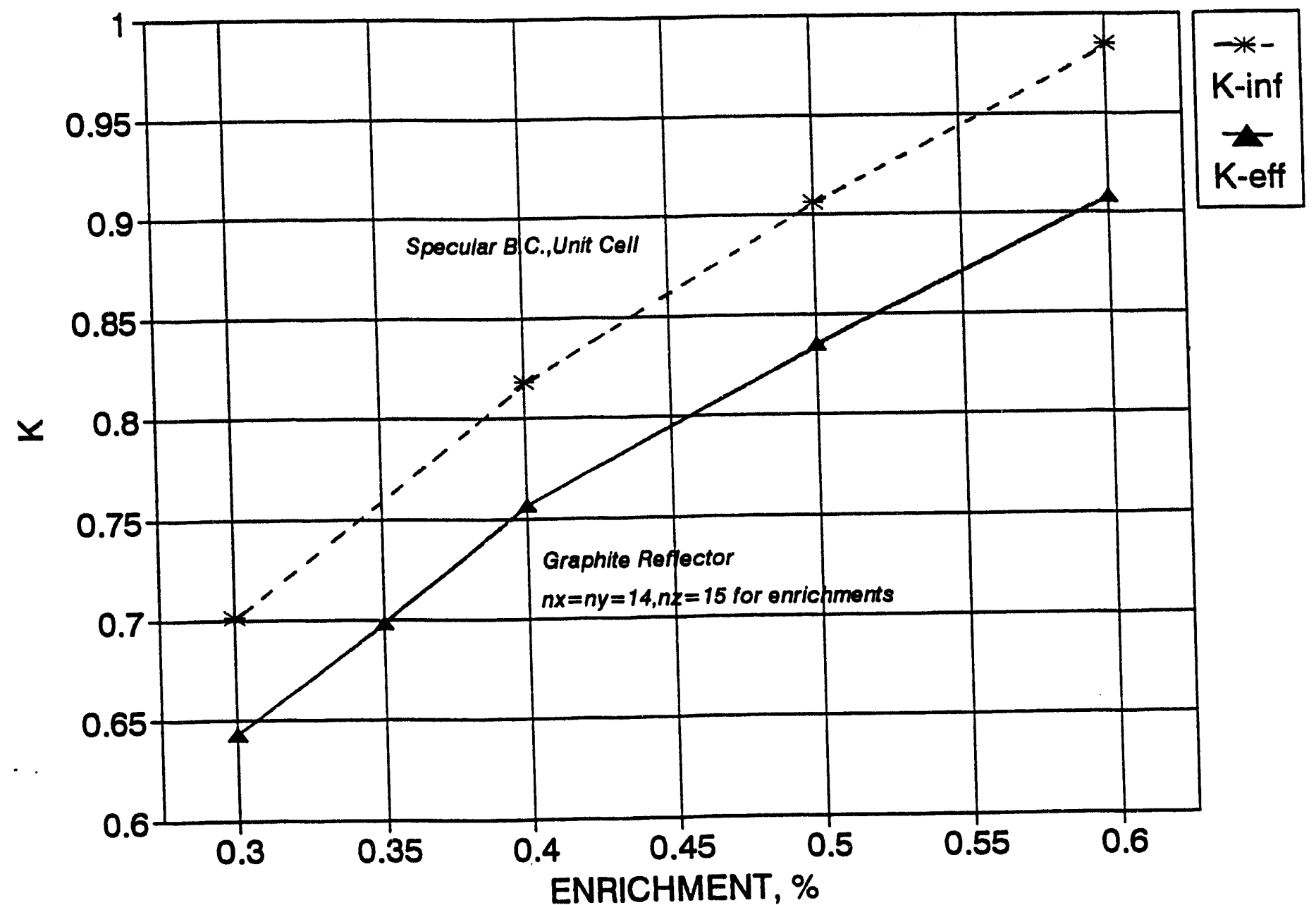

Fig 7. K VS ENRICHMENT, Discrete Slug Array, Graphite Moderator 


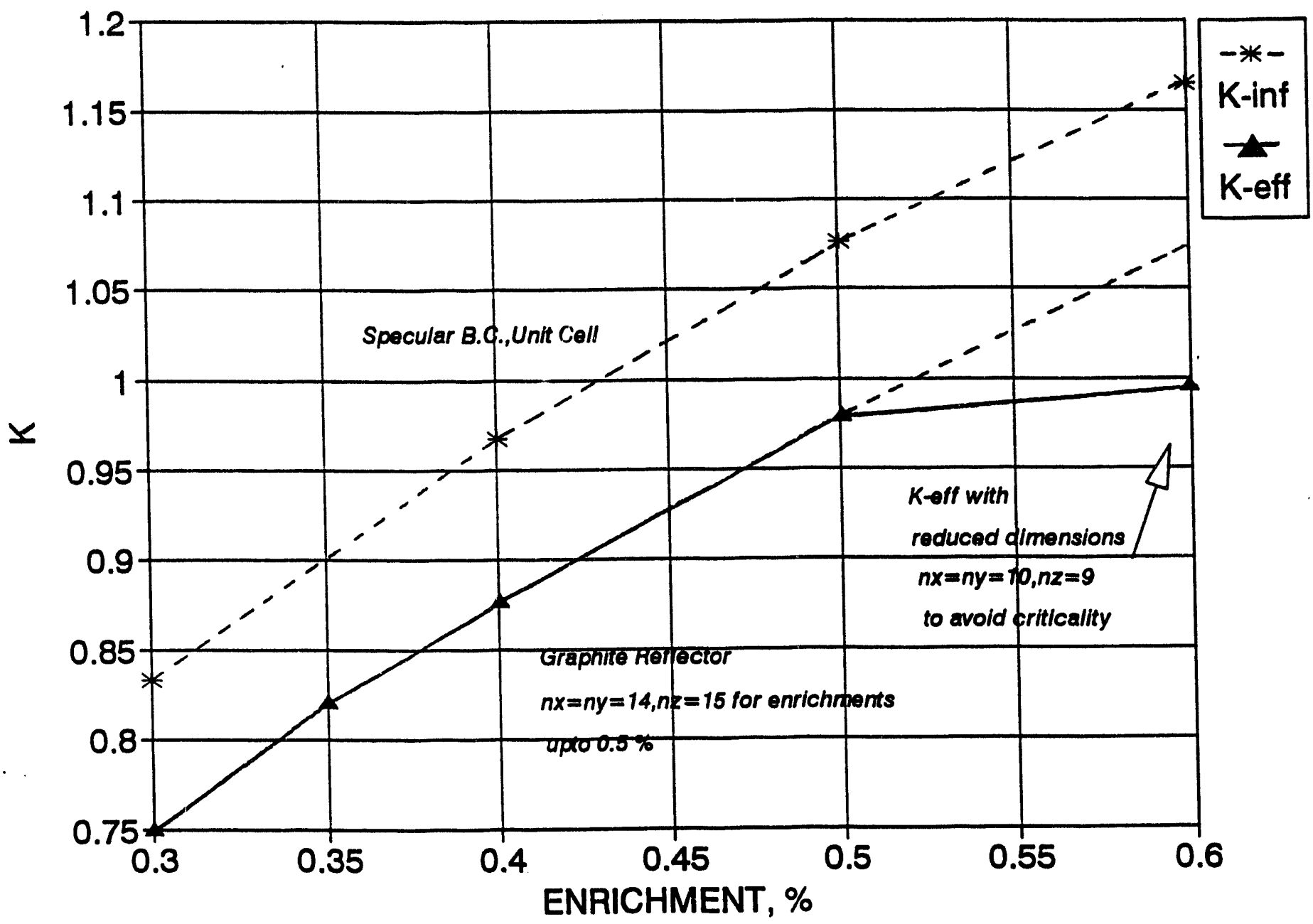

Fig 8. K VS ENRICHMENT, Discrete Slug Array, $D_{2} O$ Moderator 


\subsubsection{Alternative Moderators}

An important consideration in the design of a nuclear reactor is the control of the number and speed of the neutrons. Therefore, it is apparent that the designer must have knowledge of the effectiveness of various materials in slowing down neutrons or in capturing them. The slowing-down power depends not only on the relative energy loss per atomic collision but also on the number of collisions per second per unit volume. The former will be larger (the lower the atomic weight) and the latter will also be larger, depending on a greater atomic density and the probability of a scattering collision. The effectiveness of a moderator is frequently expressed in terms of the moderating ratio, which is the ratio of the slowing down power to the capture cross section. The values of the moderating ratios of the alternative moderators which were evaluated are presented in Table 2.

Both graphite and heavy water were used in the design of the baseline cores. However, other moderators were also tested for evaluating maximum $\mathrm{K}_{\mathrm{eff}}$ as a part of the design process by using unit cells based on the body centered cubic design employed in the baseline design study.

The moderators considered are :

1. Beryllium and Beryllium Oxide were used with a $0.3 \%$ assay to obtain maximum 
$K_{\text {off }}=0.69$ for pitch $=10.2 \mathrm{~cm}$; see Fig 9.

2. Liquid Oxygen was used with a $0.3 \%$ assay to obtain maximum $\mathrm{K}_{\text {off }}=0.69$ for pitch = $10.2 \mathrm{~cm}$; see Fig 10 .

3. Liquid ( $130 \%$ dense) Helium was used with a $0.3 \%$ assay to obtain a $\mathrm{K}_{\text {of }}=0.82$ for pitch $=70 \mathrm{~cm}$; see Fig 11 .

Finally, a comparison of the alternative moderators is shown in Fig 12.

TABLE 2. MODERATING RATIOS OF MATERIALS CONSIDERED

\begin{tabular}{||l|c|}
\hline Material & Moderating Ratio \\
\hline Graphite & 192 \\
\hline Beryllium & 143 \\
\hline Beryllium Oxide & 180 \\
\hline Liquid Oxygen & - \\
\hline Helium & $\propto$ \\
\hline
\end{tabular}




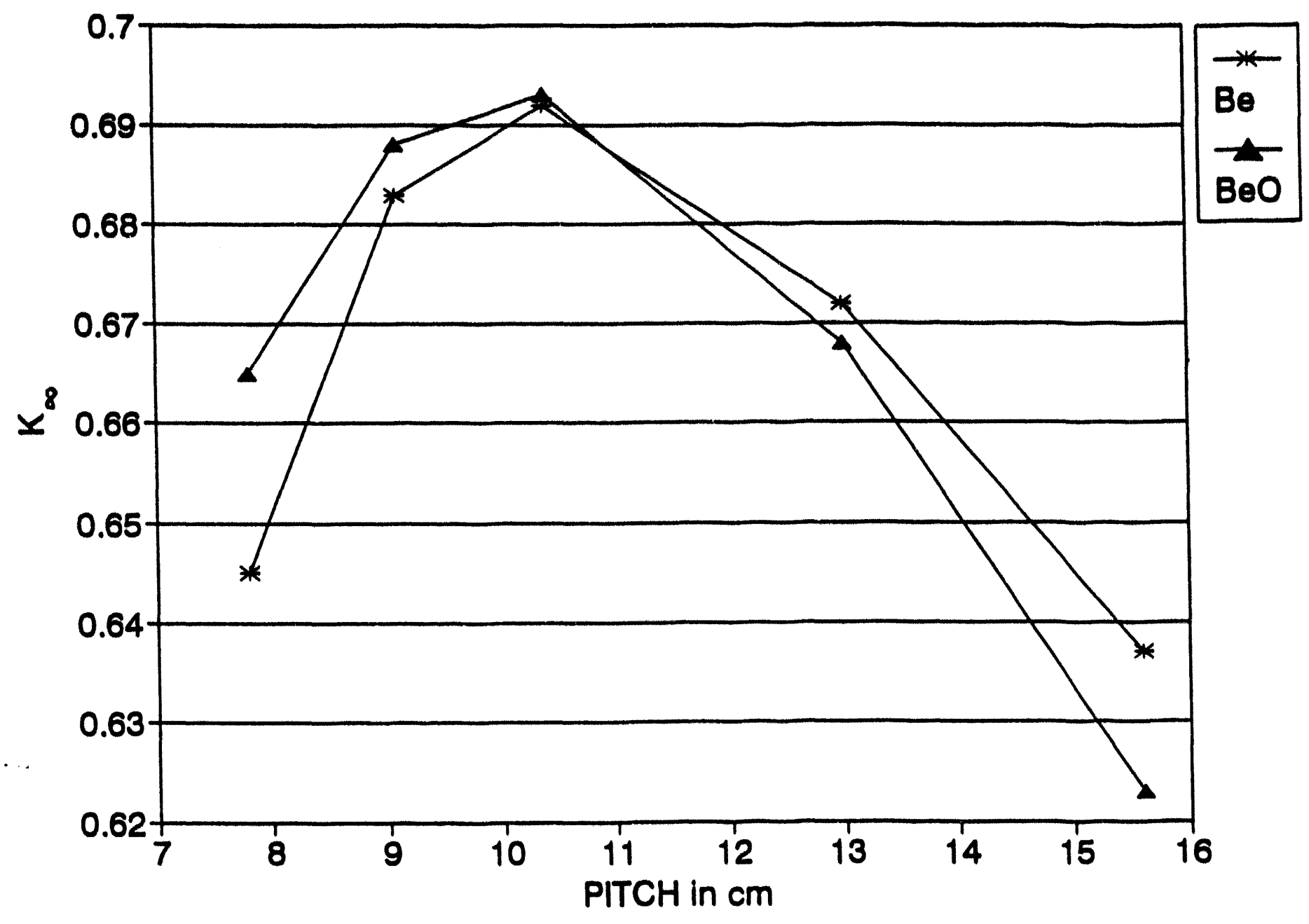

FIg 9 . Ko VS PITCH, MODERATORS BE AND BEO 


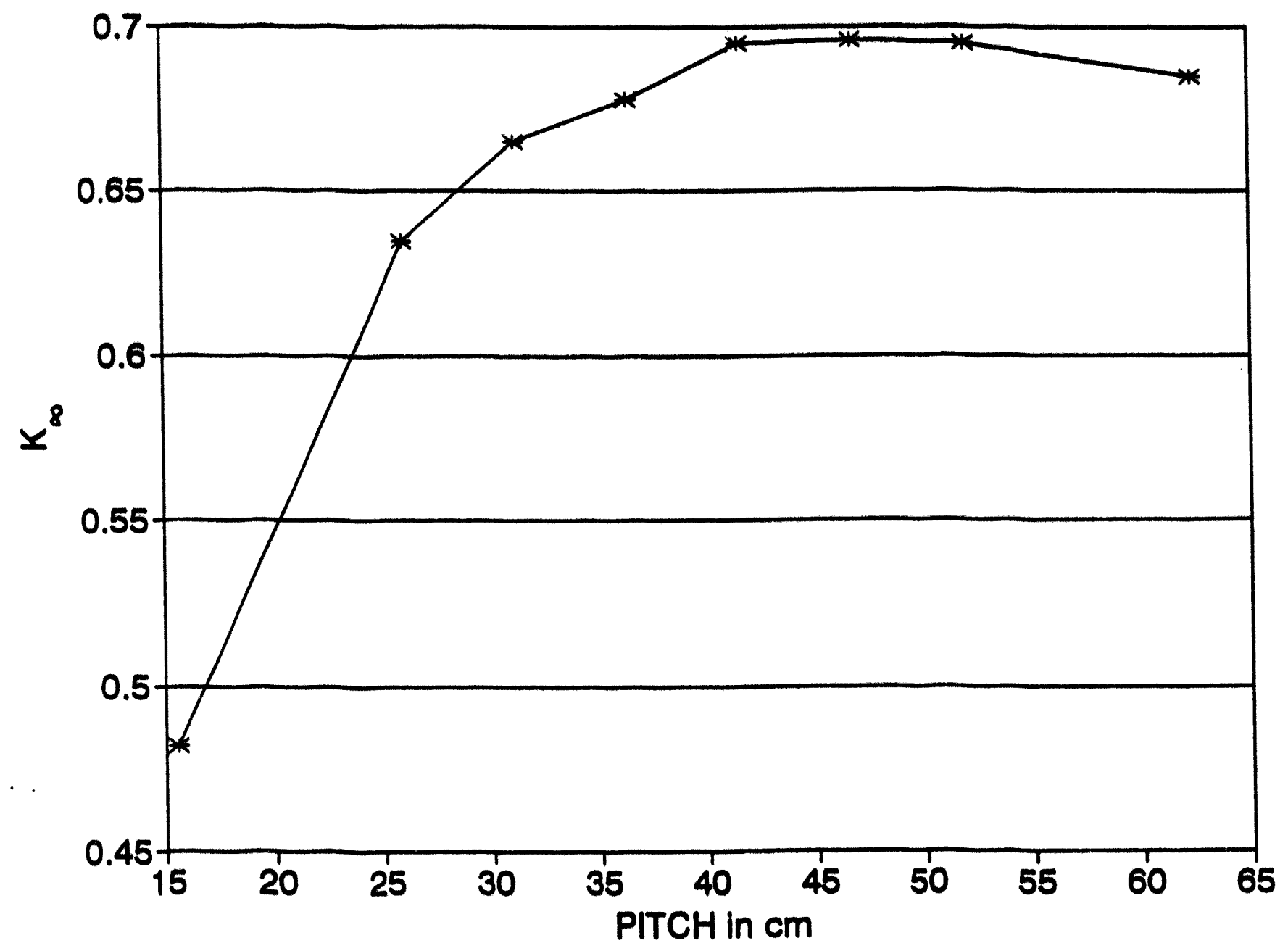

FIg 10. $K_{\infty}$ VS PITCH, LIQUID OXYGEN MODERATOR 


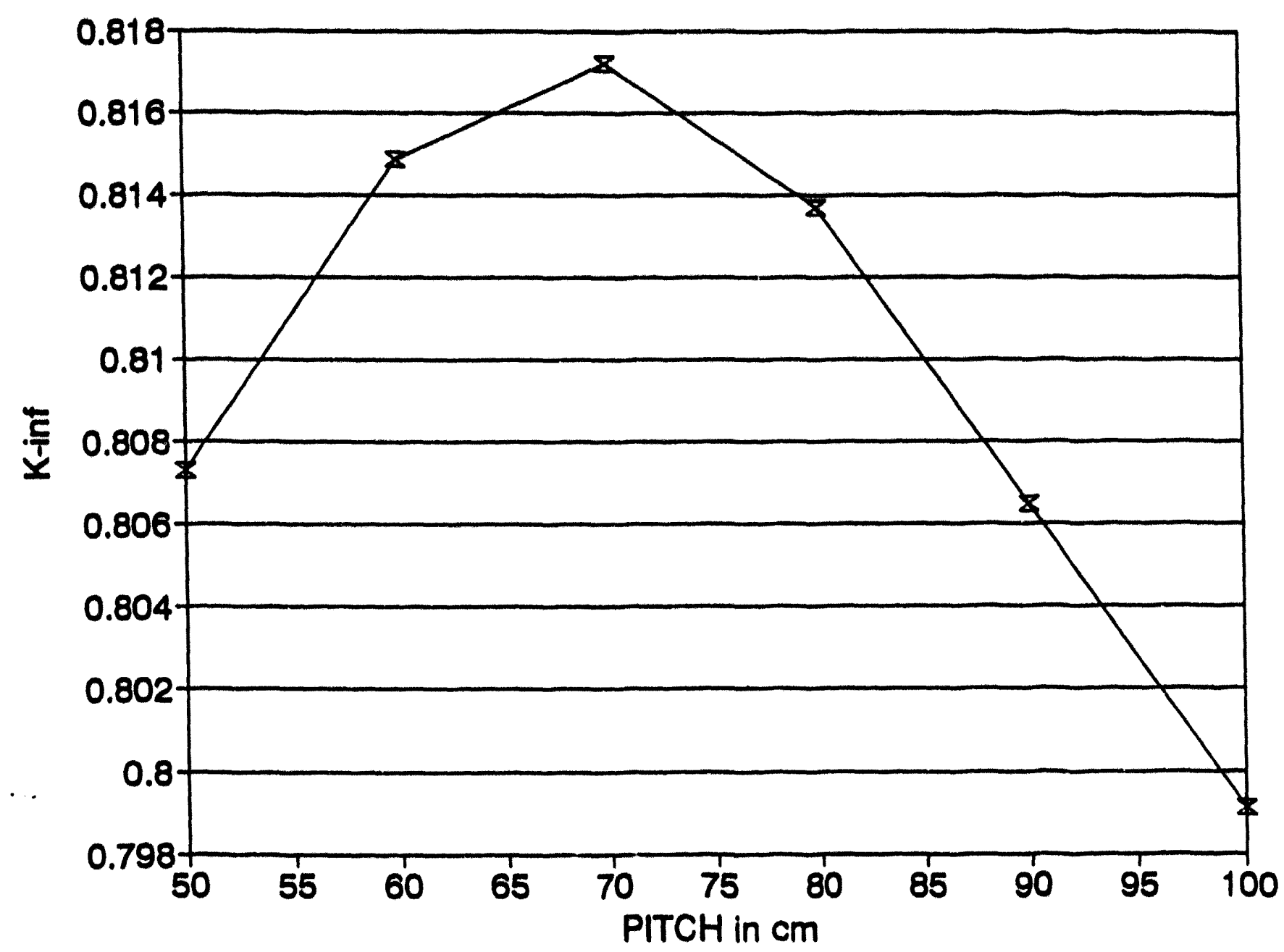

Fig 11. $K_{\infty}$ VS PITCH, MODERATOR LIQUID HELIUM 


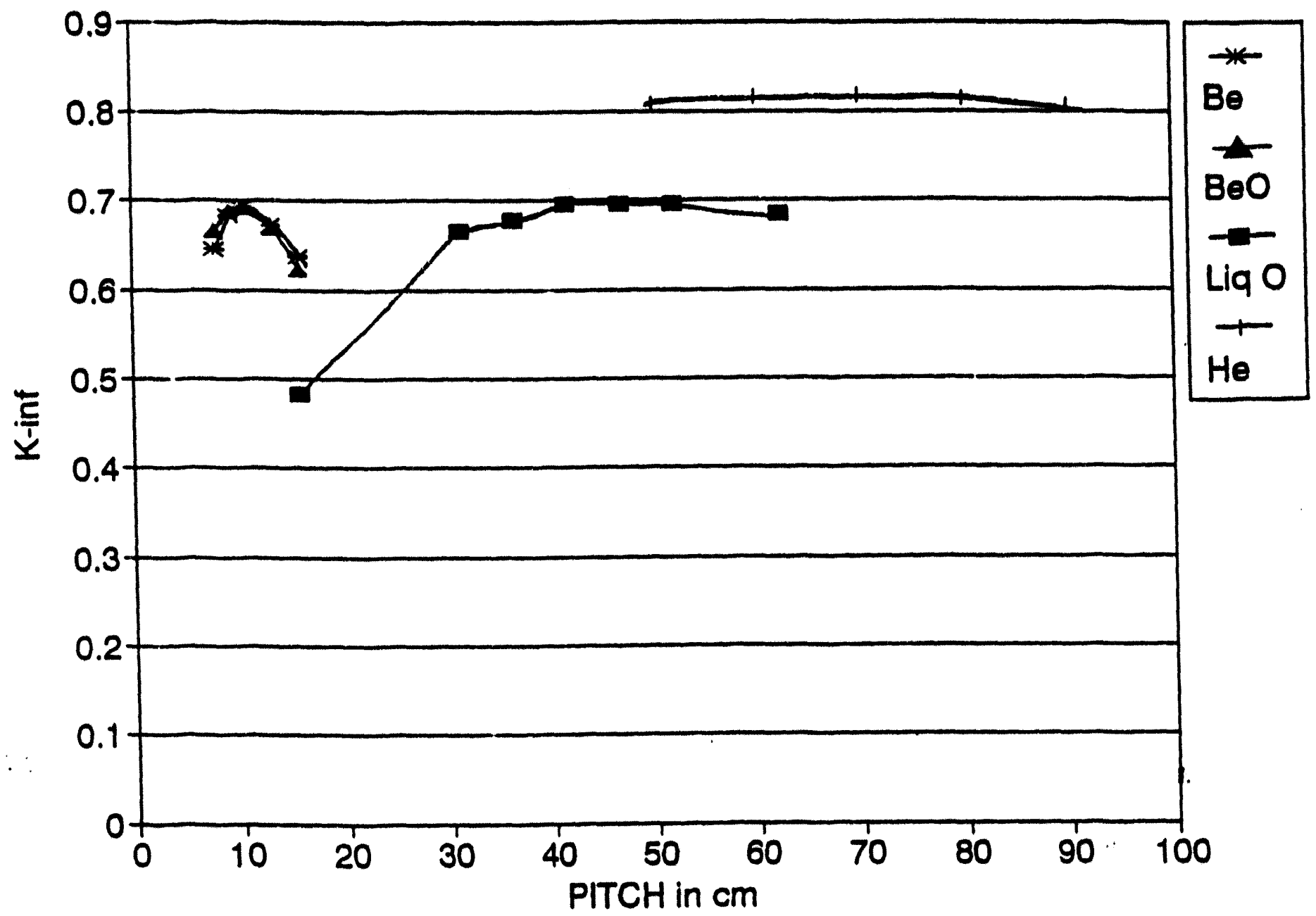

Fig 12. A COMPARISON OF ALTERNATIVE MODERATORS : Be,BeO,Liq He \& O 


\subsubsection{Reflectors}

A reflector serves to reflect back some of the neutrons escaping from the core and thereby allows them further opportunity to cause fission. The combination of materials to be used in a reflector depends, of course, upon the usual requirements of material compatibility, economy, strength, shielding, etc. On the basis of neutron conservation alone, there are two major benefits to be obtained from the addition of reflectors:

1- Neutrons escaping from the core are reduced

2- Neutrons are moderated in the reflector

In our design, after finding the optimum pitch ,we tried to find the best reflectors among graphite, concrete $\left(12^{\prime \prime}\right)$, concrete $\left(24^{\prime \prime}\right)$, water $\left(12^{\prime \prime}\right)$, and polyethylene $(\mathrm{CH})$. We found graphite to be the best reflector and used graphite as a reflector in all baseline design work.

Deuterated moderators were not considered for use as reflectors since core leakage was determined to be only modest and the use of an expensive and/or difficult-to-obtain reflector material such as deuterium is therefore unwarranted.

\subsection{Cladding poisoning}

The effect on $\mathrm{k}_{\alpha}$ was determined for the case in which the aluminum clad surrounding the uranium in the fuel slugs is removed and is shown in Fig 13.

Aluminum has 0.23 barns absorption cross section at thermal energies. Therefore it's effect on the reactivity of the system is negative. 


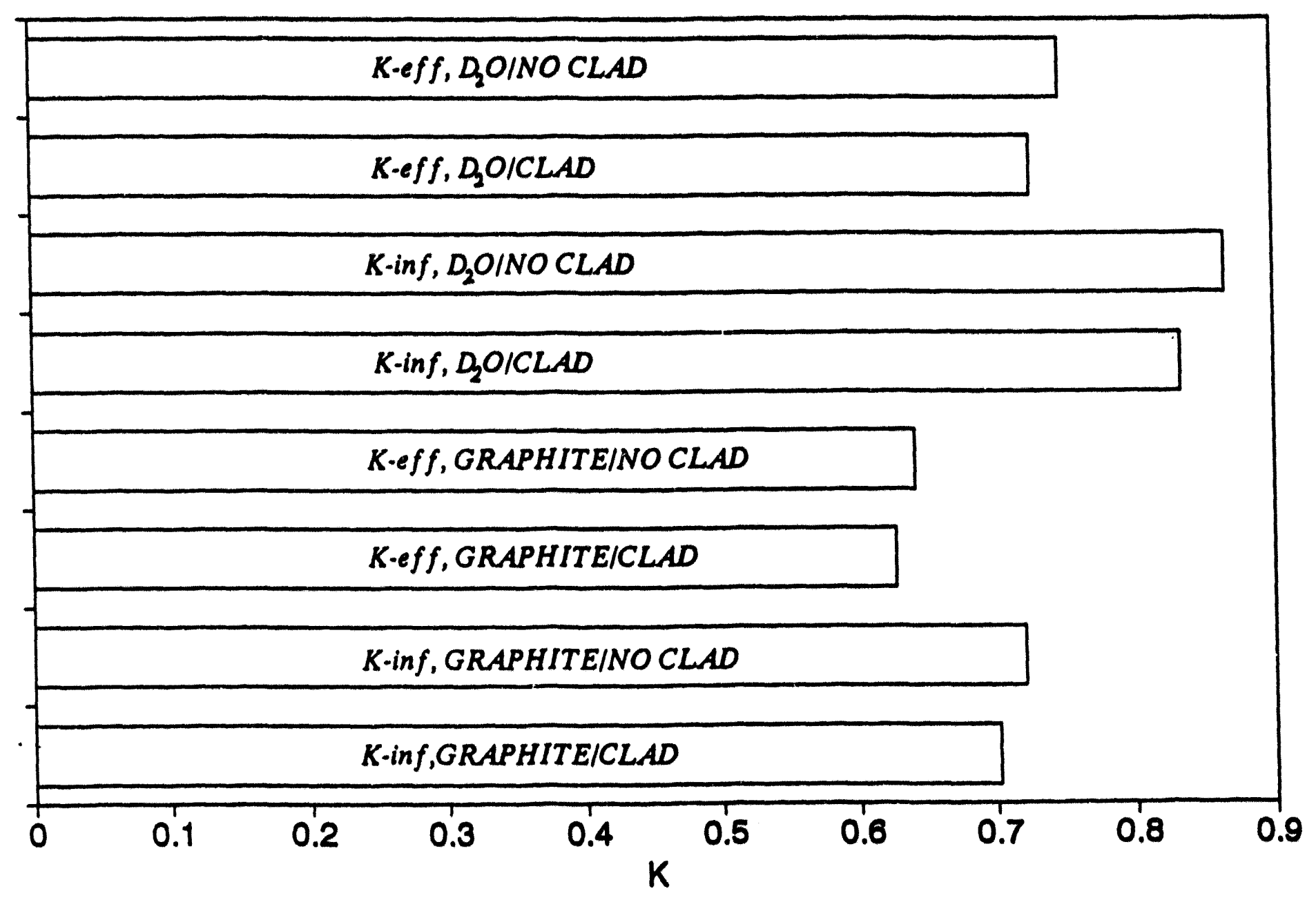

Fig 13.EFFECT OF CLADDING POISONING AT 0.3\% ASSAY 


\subsubsection{Temperature/Multiplication Factor Relationships}

Examination of graphical compilations of the cross sections of U235 show that fission cross section increases with decreasing temperature below the thermal region. This suggests the possibility that, if fission cross section increases more steeply with decreasing temperature than U238 and noderator absorptions, higher multiplication factors than predicted by thermal cross sections might be realized by simply operating a pile at cryogenic temperatures. Unfortunately, there is sparse data on cryogenic cross sections in the literature and no data in the cross section libraries commonly used for Monte Carlo calculations.

However, if recourse is again made to the idealized four factor formula (i.e. unity resonance escape probability and no capture in the moderator) coupled with a curve fit of the existing low temperature fission cross-section data for U235 of the form

$$
0,=\exp \{-0.569(\ln E)+4.269\} \text { and a } 1 / v(0 @ 0.025 e V=2.71 b) \text { relationship for }
$$

U238 absorption cross section of the form $o_{a}=0.428 / E^{1 / 2}$, an equation (equation 2) predicting low temperature $k_{\alpha}$ can be developed.

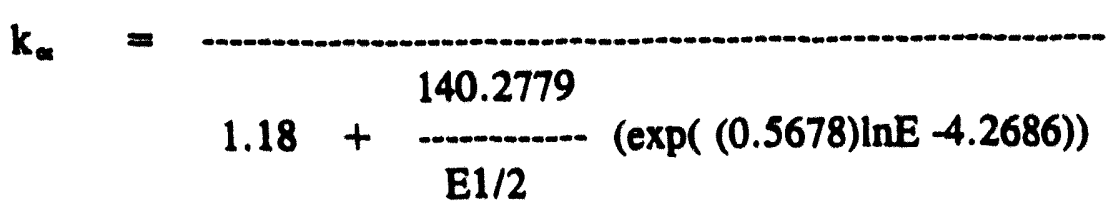


Fig 14 is a graphical presentation of equation 2 over the temperature range of 5K to 293K for fuel with an assay of $0.3 \%$. Comparison of multiplication factors predicted by equation 2 and the $k_{a}$ of 0.936 predicted by equation 1 for this fuel assay suggest the possibility of significant improvements in core multiplication at cryogenic temperatures. However, at the time of this writing, sufficient low temperature cross section data bad not been collected with which to make more sophisticated calculations. 


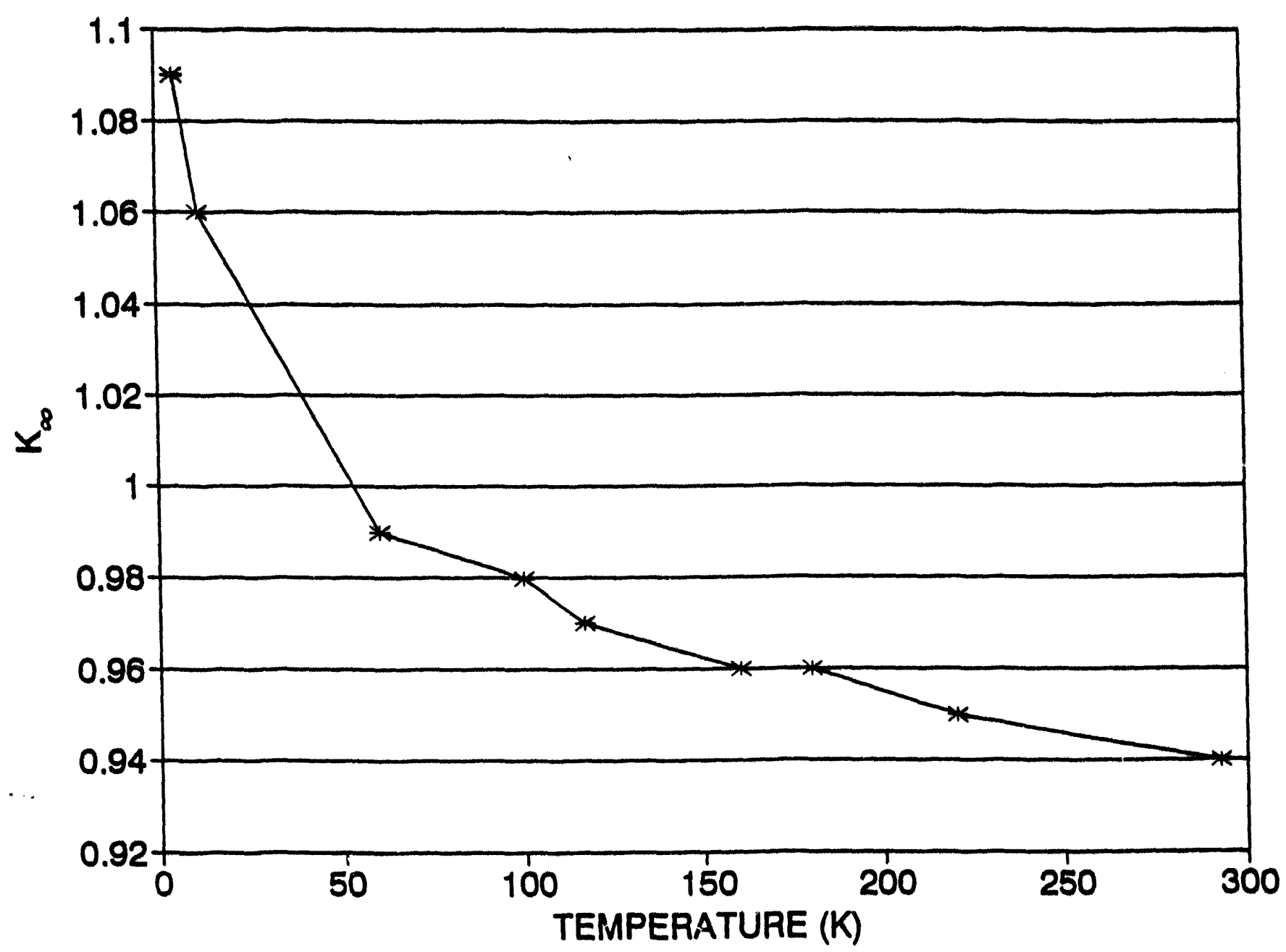

Fig 14. $\mathrm{K}_{\infty}$ VS TEMPERATURE @ 0.3\% ASSAY AS PER EQN 2 


\subsection{Conclusions}

The absence of definitive asiay data makes recommendation of a single reactor design difficult. However, it can be said with some certainty that subcritical assemblies composed of the fuel slugs and reactor grade graphite currently in the possession of the University of Tennessee Nuclear Engineering Department can be constructed so as to have multiplication factors in the range of 0.5 to 0.9 . If heavy water is used for moderation, care must be taken to avoid criticality at the upper end of the range of possible assays.

Of the designs examined to date, the one which provides the optimum balance between multiplication factor, availability of materials, ease of construction, and cost is a body centered cubic unit cell arrangement of existing fuel slugs on optimum pitch (approximately $22 \mathrm{~cm}$ for graphite moderation) placed in a $14 \times 14 \times 15$ cell cuboid with a minimum of two meters of graphite reflector. For all assays considered, the entire available fuel inventory would be used to maximize $\mathrm{k}_{\mathrm{eff}}$. Although no other readily available moderator has been identified from which a practical system could be consiructed, heavy water, if available, could greatly increase the multiplication over that achievable with graphite. In fact, with heavy water moderation and $0.6 \%$ fissile content, the highest assay for which calculations were performed, core size would be limited to $10 \times 10 \times 9$ cells to assure a subcritical multiplication factor. 


\subsection{Recommendations for Future Work}

Of paramount importance for the productive continuation of this design effort is the determination of the statistics (mean and variance) of the assay of the fuel inventory. The present agreement with the DOE facility at Oak Ridge will, if executed as planned, provide this information.

More sophisticated core modeling should also be undertaken to attempt to improve nonleakage probability with a core geometry which more closely approximates a sphere. Also, modest increases in $\mathrm{k}_{\text {eff }}$ might be realized from the improved moderator usage realized from the other lattice geometries which should be examined such as face centered cubic and hexagonal close packed.

The benefits which derive from the use of heavy water are so great that substantial effort should be put forth to obtain, on loan, some quantity of this material. Hybrid systems which have separate regions moderated by graphite and heavy water should also be analyzed.

Finally, the potential benefits of operating a graphite pile at cryogenic temperatures warrant further examination of the low temperature cross sections for the core nuclides. At a minimum, an extensive review of the literature on this subject is in order. 


$$
\begin{aligned}
& \text { Appenlix A } \\
& \text { Input Deck for rol lattice unit cell }
\end{aligned}
$$

$=c 8 a s 25$

crbr mod cyl 293k sqp ptch cell

27groupndf 4 latticeceli

uranium 1 den $=18.761293922350 .39223899 .7$ end

C 21.0293 end

at 31.0293 end

end comp

triangpitch 18.02 .6123 .03 end

read param tme $=2.0$

end param

read geom

unit 1

zhemicyl-y 111 1.3 2p102.025

zhemicyl-y 311 1.5 2p102.225

unit 2

zhemicyl+y $11121.3 \quad 2 \mathrm{p} 102.025$

zhemicyl+y $3111.5 \quad 2$ p102.225

unit 3

zhemicyl-x $1111.3 \quad 2 p 102.025$

zhemicyl-x 311.5 2p102.225

unit 5

zhemicyl+x $1111.3 \quad 2 p 102.025$ origin $-9.0 \quad 0.0$

zhemicy $1+x \quad 311.52$ p102.225 origin -9.00 .0$

cuboid 2 1 2p9.0 2p15.588 2p302.225

hole 10.015 .5880 .0

hole $20.0-15.588 \quad 0.0$

hole 39.00 .00 .0

end geom

read array nux $=1$ nuy $=1$ nuz $=1$ fill $f 5$ end array

read bnds $x y f=s p e c$ $z f=\operatorname{concl} 2$ end bnds

end data

end

$\$$ 
Appendix A $($ conti)
Input Deck for Bady ceatored cobic Lattice

a $=\operatorname{ceas25}$

crbn mod cyl 293k sqp ptch cell

27groupndf4 latticeceli

uranium 1 den=18.76 1293922350.39223899 .7 end

C 21.0293 end

al 31.0293 end

end comp

sphtriangp 18.04 .814125 .2143 end

read param tme $=2.0$

end param

read geom

unit 1

zhemicyl+x $1111.3 \quad 2$ p5.5

zhemicy $1+x \quad 3 \quad 1 \quad 1.5 \quad 2 p 5.7$

unit 2

zhemicyl-y 111 1.3 2p9.0

hole $50.0 \quad 0.0 \quad 0.0$

zhemicyl-y $3 \quad 1 \quad 1.5 \quad 2 p 9.0$

unit 3

zhemicyl-x $1111.3 \quad 2 p 5.5$

zhemicyl-x 311 1.5 2p5.7

unit 4

zhemicyl+y $11121.3 \quad 2 p 9.0$

hole $6 \quad 0.0 \quad 0.0 \quad 0.0$

zhemicyl+y 311 1.5 2p9.0

unit 5

zhemicyl-y $211.2995 \quad 2 p 3.7$

unit 6

zhemicyl+y 211.2995 2p3.7

unit 7

cuboid 2 1 2pg.0 2pg.0 2pg.0

hole $1-9.0 \quad 0.0 \quad 0.0$

hole $20.0 \quad 9.0 \quad 0.0$

hole $3 \quad 9.0 \quad 0.0 \quad 0.0$

hole $4 \quad 0.0-9.0 \quad 0.0$

end geom

read array nux $=1$ nuy $=1$ nuz $=1$ f11l $f 7$ end array

read bnds all=spec end bnds

end data

end

\$ 


\section{REFERTNCES}

Foster A.R., Wright R.L.: Basic Nuclear Engineering; Allyn and Bacon, 1983.

Glasstone S., Sesonske A.: Nuclear Reactor Engineering; Van Nostrand Reinhold Comp., 1981.

Duderstadt J.J., Hamilton L.J.: Nuclear Reactor Analysis; John Wiley

\& Sons, 1976.

Henry A.F.: Nuclear-Reactor Analysis; The MIT Press, 1975.

Lamarsh J.R.: Introduction to Nuclear Reactor Theory; Addison-Wesley Publ. Comp., 1966.

Parks C.V.: Scale-4: An Improved Computational System for Spent-Fuel Cask Analysis;

Proceedings of the 9 th International Symposium on the Transportation of Radioactive Materials, Washington, DC, June 11-16, 1989.

SCALE: A Modular Code System for Performing Standardized Computer Analyses for Licensing Evaluation, Vols. 1-3; NUREG/CR-0200, U.S. Nuclear Regulatory Commission, Revision 3, December 1984.

Zweifel P.F.: Reactor Physics; McGraw-Hill Book Comp., 1973.

Robert V. Meghreblian: Reactor Analysis; McGraw-Hill Book comp., 1960 
Distribution:

The University of Tennessee

Nuclear Encineerine Department

H. L. Dodds

Qak Ridre Y-12 Plant

E. L. Chumetski

A. Lo/DOE-OST, 9731, MS-8175 (2)

Y-12 Central Files - RC 

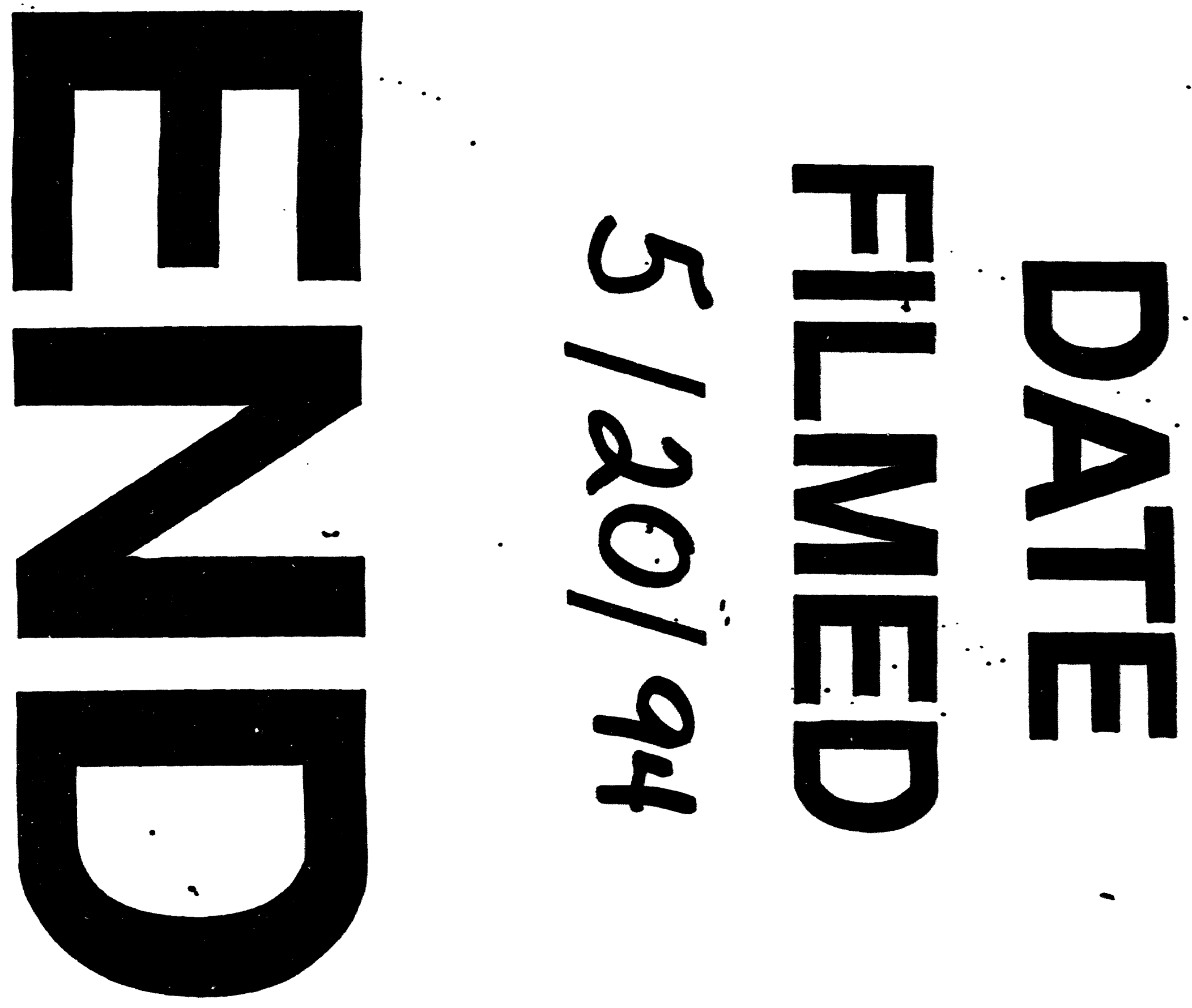
